\title{
Comb Type Pilot Based Channel Estimation using Dft Based Interpolation for Spatial Modulated OFDM Systems
}

\author{
Anetha Mary Soman, R Nakkeeran, Shinu Mathew John
}

\begin{abstract}
An integration of Spatial Modulation with Orthogonal Frequency Division Multiplexing (SM OFDM) is a recently evolved transmission technique. In practical scenarios, channel estimation is significant for detecting transmitted data coherently. Impulse response based interpolation technique that provides channel frequency response estimate with reduction in noise error is proposed for comb type pilot based channel estimation of SM OFDM system along with $1 D$ interpolation techniques under frequency selective channel. This scheme focus on carrying out smoothing and estimation in time domain and transforming output back to the frequency domain. BER performance is investigated for Rayleigh channel employing COST 207 project model on two test urban environments (Typical and Bad) for 4 and 16 QAM SM OFDM systems. Results show that the Least Square estimator with DFT interpolation performs finer compared to all one dimensional interpolation methods with less computational complexity by employing FFT algorithms.
\end{abstract}

Keywords: Multiple Input Multiple output (MIMO), Multicarrier modulation, Spatial Modulation, Channel Estimation, Interpolation.

\section{INTRODUCTION}

$\mathrm{H}_{\mathrm{i}}$ igh data rates, spectral efficiency, low complexity and flexibility are some of the requirements of upcoming wireless communication systems. MIMO technology has emerged as a favored aspirant in the account of these measures [1]. OFDM, a multicarrier modulation technique, transforms a frequency selective channel to several parallel flat sub channels. Combining OFDM with MIMO technique increases spectral efficiency and improve link reliability [25]. Spatial modulation (SM), a space modulation technology is a new low complexity data throughput enhancing technique introduced by Meshleh. $\mathrm{R}$ and Haas. $\mathrm{H}$ in the year 2006 [6] and remains as a unique single stream MIMO transmission technique. The index of one of the available antennas being active avoids Inter Channel Interference (ICI). SM-OFDM system model, an alternative MIMO technique is analyzed in this paper [7].

Manuscript received on March 23, 2021.

Revised Manuscript received on June 23, 2021.

Manuscript published on June 30, 2021.

* Correspondence Author

Anetha Mary Soman, Research Scholar, Department of Electronics Engineering, School of Engineering and Technology, Pondicherry Central University, Pondicherry, India

R Nakkeeran, Associate Professor, Department of Electronics Engineering, School of Engineering and Technology, Pondicherry Central University, Pondicherry, India.

Shinu Mathew John, Professor, Department of Computer Science \& Engineering, St. Thomas College of Engineering \& Technology, Kannur (Kerala), India.

(c) The Authors. Published by Blue Eyes Intelligence Engineering and Sciences Publication (BEIESP). This is an open access article under the CC BY-NC-ND license (http://creativecommons.org/licenses/by-nc-nd/4.0/)
Based on SM mapper, multiple information bits are mapped into single information symbol and a corresponding antenna number. Selected transmit antenna involves in transmission and other antennas remain silent for corresponding sub channel and time instant. At the receiver side, for each subcarrier, estimation of symbol and active antenna number takes place and original information bits are demapped.The transmitted bits depends on a constellation diagram and the number of antennas used for transmission. [8]. Ninety percentage complexity reductions are obtained by integrating SM in OFDM.

Perfect channel state information is always impractical and hence estimation of channel is of vital importance. Estimation of channel is an integral part of receiver design. It is a challenging issue in wireless systems. The channel response varies over time because of the movement of transmitters, receivers and scattering objects. Therefore, the estimation of channel is important for SMOFDM systems. For single carrier SM systems, estimation of the channel has been considered in [9] [10]. Pilot based channel estimate where pilots are added along with the symbols carrying information [11] is commonly used to obtain channel state information (CSI) at the receiver. Iterative channel estimation based detection systems are proposed in [12] Estimation of channel for coherent SM OFDM systems relies on pilot sequence adapted and channel characteristics. Channel estimation based on pilot tones is one among favorable methods for frequency selective channels [13]. Few subcarriers are used in the pilot mode for the initial estimation process. Estimation techniques are based on frequency and time domain techniques. Initially to find the channel estimate on pilot tones, least squares (LS) algorithm is applied in the frequency domain. The frequency response for the known pilot subcarrier is estimated as an equation.

$$
\hat{H}_{L S}=\operatorname{diag}\left(X_{1}\right)^{-1} Y_{1}+\operatorname{diag}\left(X_{1}\right)^{-1} W_{1}
$$

$\mathrm{Y}_{1}$ is the received samples, $\mathrm{H}$ is channel matrix, $\mathrm{W}_{1}$ is the noise component at each subcarrier for $\mathrm{n}^{\text {th }}$ OFDM symbol and $\mathrm{X}_{1}$ is the input data. Using interpolation, the estimates on all subcarriers can be found. The interpolation process is denoted as

$$
\hat{H}=Q \hat{H}_{L S}
$$

Where Q represents the interpolation matrix. The aim of the channel estimation technique is to acquire $\mathrm{Q}$ with less computation and to attain greater accuracy for a given system.

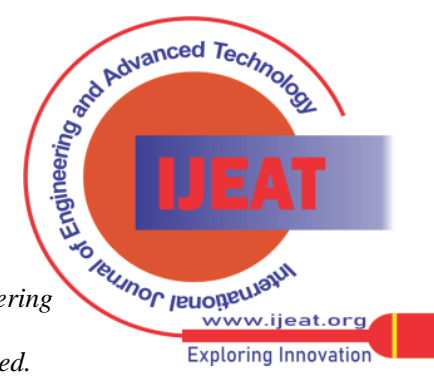


This paper discusses channel estimation using Discrete Fourier Transform (DFT) in addition to one dimensional interpolation methods. The DFT method can come up with acceptable results by lessening noise on estimated channel coefficients [14]. A classical comb type pilot based frame format used for MIMO is not appropriate for SMOFDM systems. Since a single antenna at transmit side is active at a time and all other antennas remain silent for each subcarrier, a new frame structure is put forward for $N_{t} \times N_{r}$ SM OFDM systems [15]. To trace frequency selectivity of the channel, pilot tones are placed precisely in the frequency domain. Interpolation methods are then used to obtain the frequency response of channel at data symbols.

The remaining paper is ordered as follows. Section II explains system model for SM OFDM Systems. Section III includes estimation of channel with description. SectionIV presents impulse response based channel estimation. Section $\mathrm{V}$ provides performance analysis and Section VI gives the conclusion.

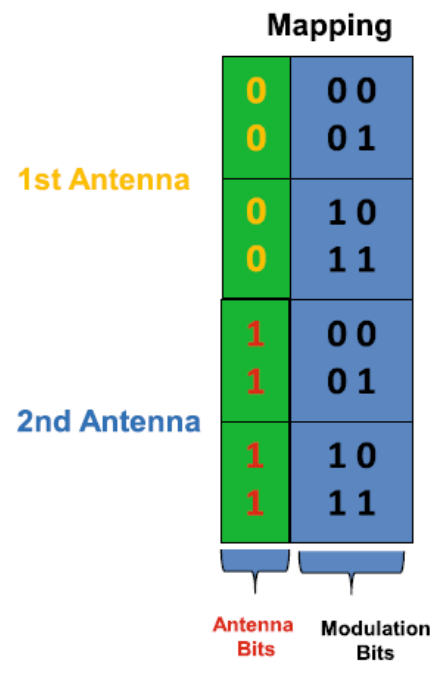

\section{SPATIAL MODULATION OFDM SYSTEM MODEL}

Let $Z(k)$ represents $d \times N$ matrix of binary data to be transmitted. From the matrix, $d$ represents the entire number of bits/symbol in a subcarrier, $N$ representsthe total number of OFDM subcarriers. Based on SM mapper represented in Fig.1 the data $Z(k)$ is mapped to a $N_{t} \times N$ matrix, $N_{t}$ represents the entire number of antennas used for transmission. In the matrix, entire elements of $X_{1}(k)$ remain zero except at mapped location of the transmitting antenna. On each OFDM sub channel, the number of bits transmitted is represented as

$$
m=\log _{2}\left(N_{t}\right)+\log _{2} M
$$

M represents the modulation degree.
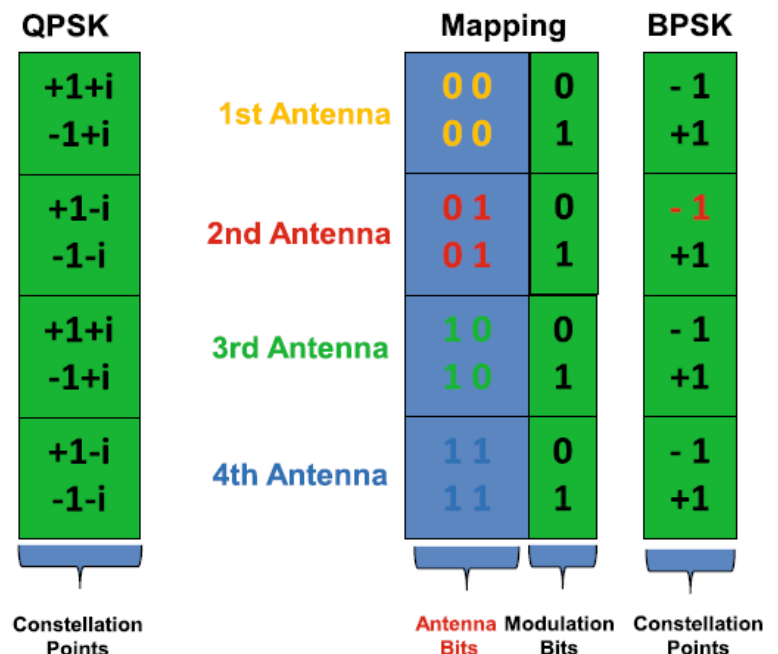

Figure 1 SM OFDM Mapper

Each row vector of $X_{1}(k)$ is dealt by an OFDM modulator where data symbols gets transformed from serial to parallel form. The data symbols in each row are named as subcarrier. If each OFDM symbol consists of $N$ data symbols, then there are $\mathrm{N}$ number of subcarriers. To transmit known data, pilot insertion is done which aid in channel estimation at receiver. Inverse Discrete Fourier Transform (IDFT) operation is carried out on each row vector of $X_{1}(k)$ and transmits from the $i^{\text {th }}$ antenna as

$X_{i}(n)=\sqrt{\frac{1}{N}} \sum_{k=0}^{N-1} X_{1 i}(k) e^{\frac{j 2 \Pi k n}{N}} 0 \leq n \leq N, 0 \leq k \leq N$ (4)

Where $N$ represents the number of FFT points. To prevent distortion caused by Inter Symbol Interference (ISI) in channel cyclic prefix (CP) is affixed at starting of each OFDM Symbol. CP is the copy of the end part of the particular OFDM symbol. The resultant OFDM symbol after the insertion of $\mathrm{CP}$ is transformed to serial form and gets passed through the channel. For multipath fading environment, the response of channel in time domain between $i^{\text {th }}$ transmitting antenna and $j^{\text {th }}$ receiving antenna is written as

$h_{i j}(n)=\sum_{l=0}^{L-1} h_{i j, l} \delta\left(n-\tau_{i j, l}\right)(5)$

Where $L$ is the number of paths, $h_{i j, l}$ is complex time varying channel coefficients and $\tau_{i j, l}$ is the delay of $l^{\text {th }}$ path.

At receiver, symbols are initially transformed to parallel form.Then CP is removed from symbols.

Frequency domain symbols are obtained by passing the OFDM symbols through DFT block and are used to estimate the channel by a pilot based equalizer. Further procedure processes the obtained data to estimate the transmitted OFDM symbols.

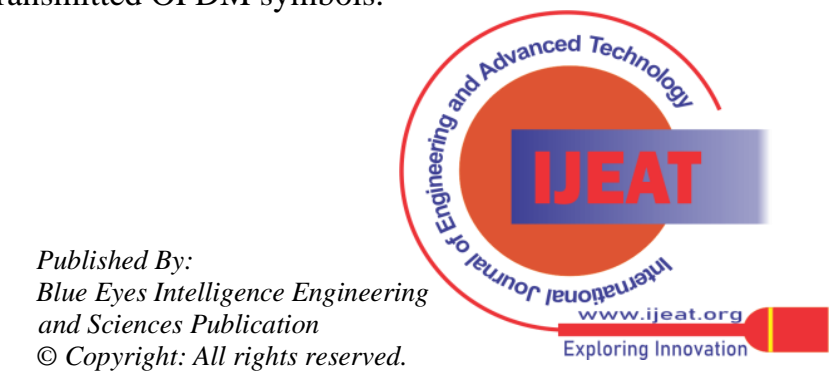


Parallel to serial conversion takes place and demapping is done such that the binary data is obtained as transmitted.
The OFDM demodulator output for $k^{\text {th }}$ subcarrier is written as
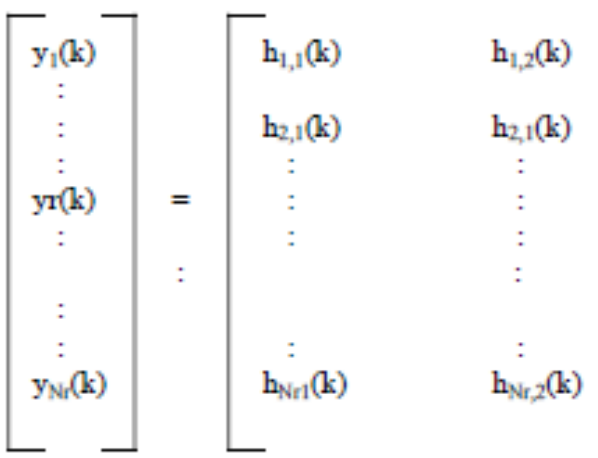

Where $h_{r j}(k)$ is the channel coefficient between transmit antenna $j$ and receive antenna $r \quad x_{q}(k)$ is $q^{\text {th }}$ active antenna symbol from constellation figure and $w_{r}(k)$ is complex valued White Gaussian Noise with variance $\sigma_{w}{ }^{2}$ and zero mean.

Matrix form representation of signal model is rewritten as

$$
\begin{array}{r}
y(k)=H(k) x_{j}(k)+w(k) k=1,2, \ldots . . N \\
x_{j}(k)=\left[0 \ldots \ldots . x_{q}(k) \ldots \ldots . .0\right]^{T} .
\end{array}
$$

In spatial modulation the detection process necessitates the finding of the transmitting antennas index and the modulation symbol send on it. For each OFDM sub channel, Maximum likelihood (ML) based detection is performed. The optimum detection rule is

$$
\begin{aligned}
& {\left[\hat{j}_{M L}, \hat{q}_{M L}\right]_{k}=\underset{j, q}{\operatorname{argmax}}\left\|h_{j}(k) x_{q}(k)\right\|_{F}^{2}-2 \operatorname{Re}\left\{y^{+}(k) h_{j}(k) x_{q}(k)\right\} \text { (8) }} \\
& \quad \text { for } 1 \leq j \leq N_{t}, 1 \leq q \leq M . x_{q}(k) \text { represents } q^{\text {th }}
\end{aligned}
$$
active antenna symbols from constellation figure, $h_{j}(k)$ represents the $j^{\text {th }}$ column of $\mathrm{H}(\mathrm{k})$.

\section{CHANNEL ESTIMATION}

Channel Estimation is defined as specifying a mathematically modeled channel. The design benchmark of estimators is to reduce mean square error (MSE) and complexity in computation. Estimation algorithms are used to find channel impulse response (CIR) or channel frequency response (CFR) in the time domain and frequency domain. This paper considers comb type pilot based

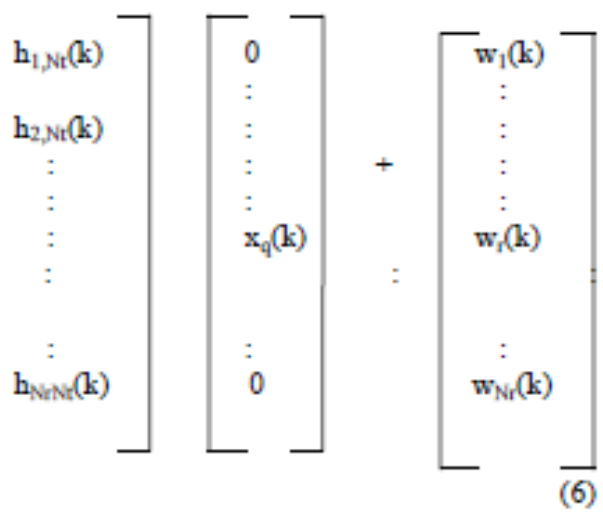

channel estimation for SM OFDM systems. As an initial step to the estimation process, pilot insertion should be taken care of. Too much pilot insertion cause spectrum overhead. So the pilot spacing should be as small as possible. This makes certain time varying, frequency selective channel to be in good track. Frame structure uses comb type pilot insertion. Pilot tones are set at the periodically located subcarriers of every OFDM symbol. To estimate the channel on frequency axis, interpolation is used.

Let $T_{f}$ represents period of pilot tones in frequency. To keep trace of channel characteristics, pilot tones must be accommodated as regularly as coherence bandwidth is. Then pilot tone period should satisfy the inequality

$$
T_{f} \leq \frac{1}{\sigma_{\text {max }}(9)}
$$

Where $\sigma_{\text {max }}$ representsa maximum delay spread.

To estimate the channel accurately, Least Square (LS) Algorithm is used to find known pilot's frequency response inserted in the transmitted frame. Interpolation is then done to estimate the frequency response of the channel for each subcarrier. For an SM-OFDM system, channel state information is required to make out modulated symbols and transmit antenna indices. Compared with the classical comb type frame format, a new one is designed for SM OFDM systems as single transmit antenna gets activated and the remaining ones transmits null for each subcarrier. Fig.2 represents the frame format. In the figure, pilot tones are placed periodically over subcarriers and for the respective OFDM symbol number, each transmit antenna pass on pilots.

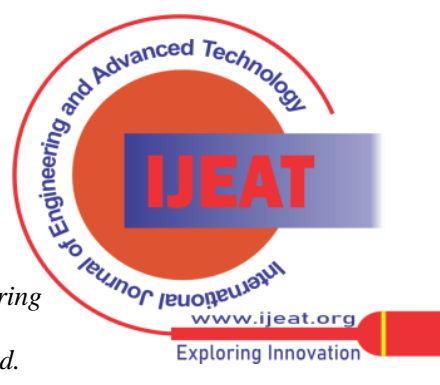




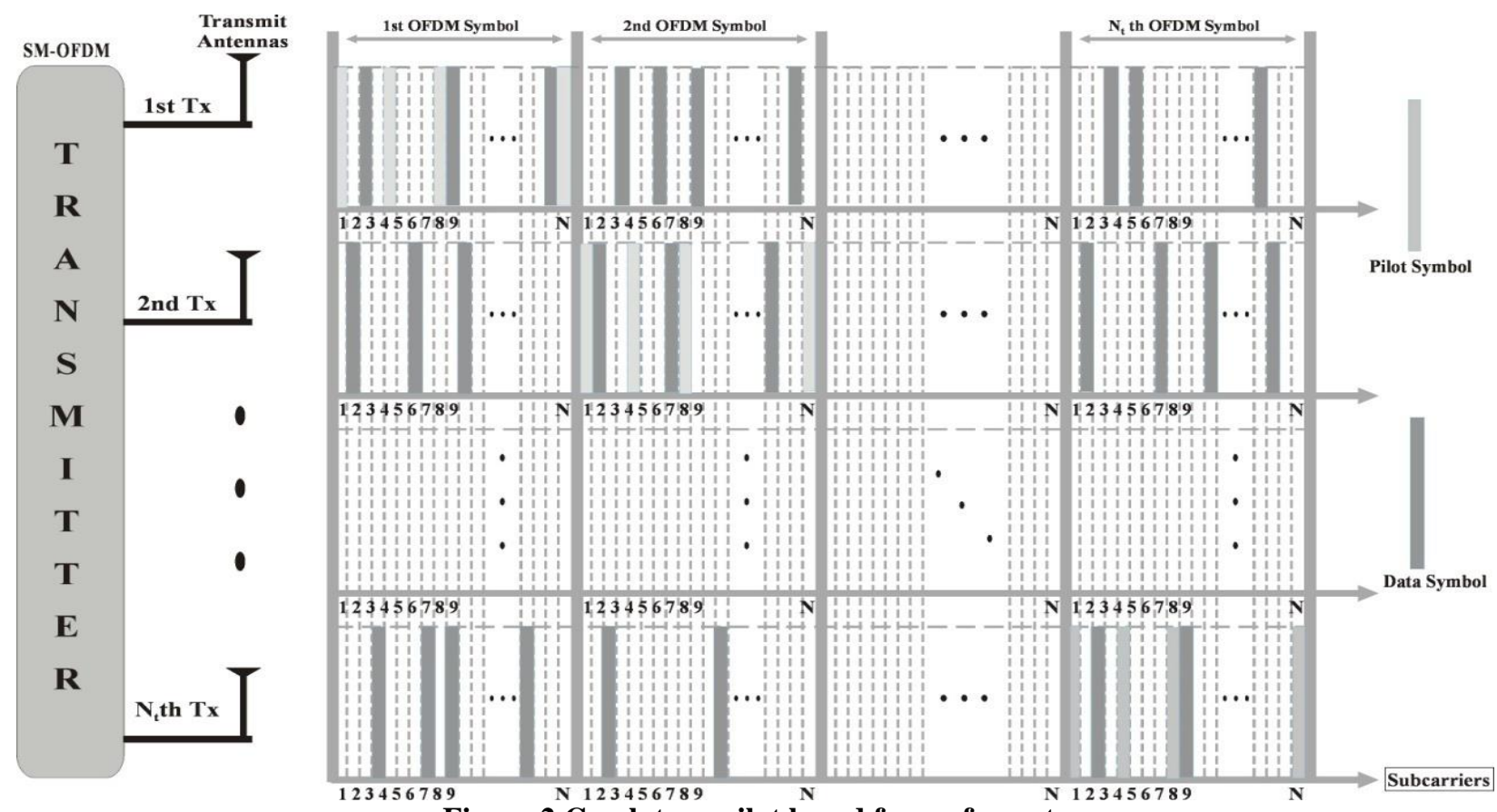

Figure 2 Comb type pilot based frame format.

An LS based channel estimator performs LS estimation at pilot positions using the retrieved and known pilot symbols. Interpolation is then performed to obtain channel frequency response at entire positions. Fig.3shows pilot aided estimator .

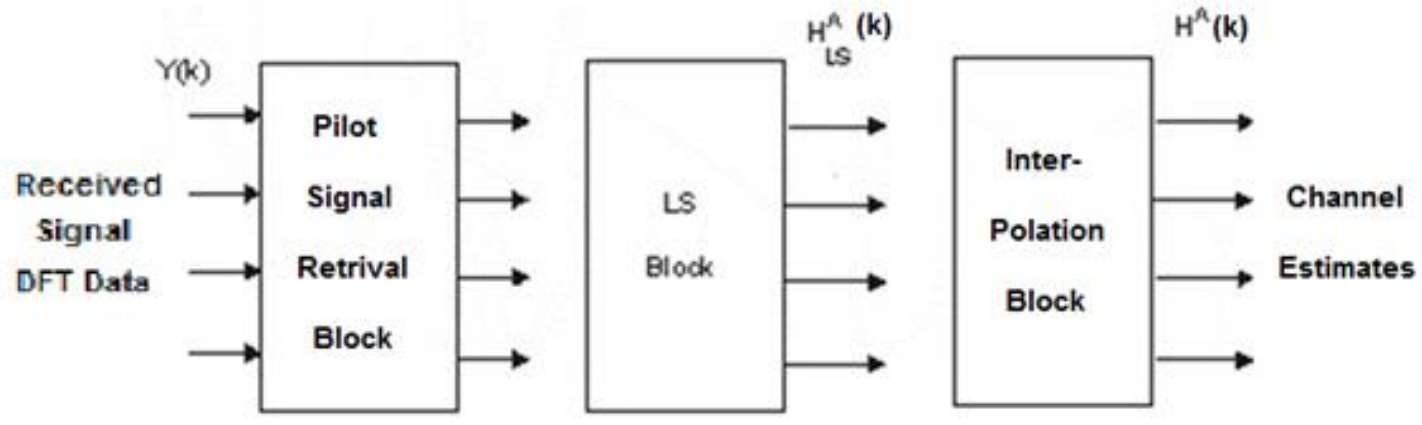

Figure 3 Pilot aided estimator

For all OFDM symbols, signals received on pilot subcarrier $k_{p}$ are represented as

$y_{i}\left(k_{p}\right)=\rho h_{i}\left(k_{p}\right)+w_{i}\left(k_{p}\right) i=1,2, \ldots . P$

Where $\rho$ represents the pilot tone, $\mathrm{P}$ represents the entire number of transmit antennas.

Channel frequency response (CFR) coefficients obtained by LS estimation are represented as

$h_{i}^{\wedge}\left(k_{p}\right)=y_{i}\left(k_{p}\right) / \rho$

Using the estimates found in pilot positions, estimate at data positions is acquired by applying different interpolation technique.

Rewriting LS estimated CFR by substituting values of $y_{i}\left(k_{p}\right)$

$h_{i}^{\wedge}\left(k_{p}\right)=h_{i}\left(k_{p}\right)+\frac{w_{i}\left(k_{p}\right)}{\rho}$ $h_{i}^{\wedge}\left(k_{p}\right)=h_{i}\left(k_{p}\right)+v_{i}\left(k_{p}\right)$ wherev $_{i}\left(k_{p}\right)=\frac{w_{i}\left(k_{p}\right)}{\rho}$

Some of the prominent interpolation techniques considered in this paper are linear interpolation, spline interpolation and low-pass interpolation and are comparatively considered by Coleri et.al [16]. In [17] Impulse response based channel estimation methods have been studied where the scheme does time domain estimation and transforms the result to frequency domain. Literature [18-20] details the Spline interpolation technique and [21] details the Low pass interpolation technique.

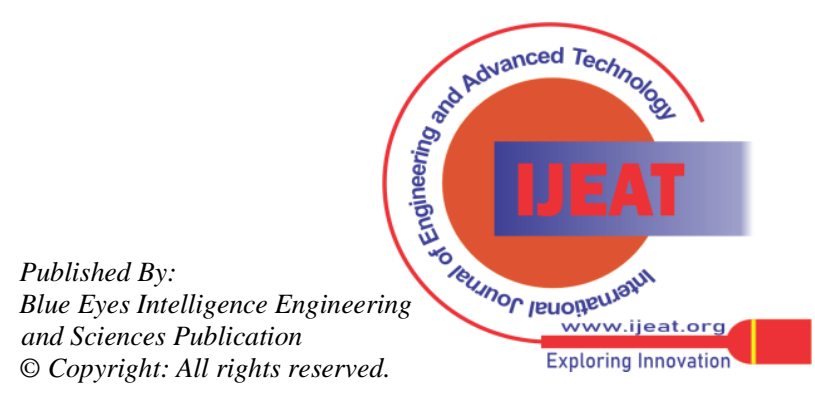




\section{IMPULSE RESPONSE BASED CHANNEL ESTIMATION}

The DFT method is considered as an impulse response based channel estimation method which provides better accuracy. It enhances performance of LS estimation by removing the noise effect in time domain outside the extreme channel delay length. The channel frequency response coefficients obtained are first transformed using IDFT.A filter is then applied in the time domain. It is assumed that extreme channel delay is within the OFDM symbol CP. These are then applied with DFT to return to frequency domain.

Mathematically, if $\hat{H}(k)$ represent estimate of channel gain at $k^{\text {th }}$ subcarrier acquired by LS estimation, then IDFT of the channel estimate $H^{\wedge}(k)_{k=0}^{N-1}$ is

IDFT $\{\hat{H}(k)\}=h(n)+w(n) \underline{\Delta} h^{\wedge}(n) n=0,1, \ldots . N-1$
Where $w(n)$ represent the noise component in time domain. Disregarding coefficients of $h^{\wedge}(n)$ which contain only noise by defining the coefficients for the maximum channel delay length $\mathrm{L}, \hat{h}_{D F T}(n)$ is written as

$$
\hat{h}_{D F T}(n)=
$$

$\left\{\begin{array}{l}h(n)+w(n) n=0,1,2 \ldots \ldots . . L-1 \\ 0 . \ldots \text { o.w }\end{array}\right.$

Finally, transforming remaining L elements to frequency domain we get

$$
\hat{H}_{D F T}[k]=D F T\left\{\hat{h}_{D F T}(n)\right\}
$$

( $\mathrm{L}$ should be known is advance)

The block diagram of channel estimator based on DFT is shown in Fig.4.

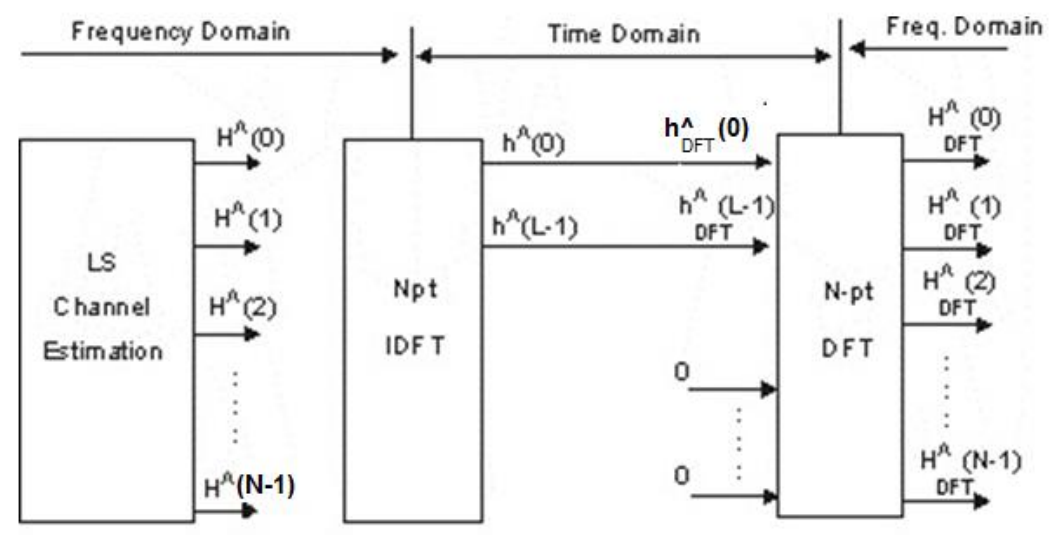

Figure 4 DFT based channel estimator

\section{PERFORMANCE ANALYSIS}

This section quantifies the bit error rate (BER) performance of $4 \times 4$ SM OFDM system under frequency selective channels. System parameters employed for the simulation are specified in Table 1

Table 1 System Parameters

\begin{tabular}{|l|l|}
\hline Parameters & Value \\
\hline Carrier frequency & $2 \mathrm{GHz}$ \\
\hline FFT size & 32 \\
\hline SNR & $30 \mathrm{~dB}$ \\
\hline Number of subcarriers & 256 \\
\hline Guard Interval & 4 \\
\hline Antenna Configuration & $4 * 4$ \\
\hline Cyclic prefix & 32 \\
\hline Channel property & $\begin{array}{l}\text { TU (Typical Urban) and BU (Bad Urban) channel model } \\
\text { based on the COST 207 Project }\end{array}$ \\
\hline Modulation Scheme & M-QAM(4,16) \\
\hline Pilot ratio & $1: 8$ \\
\hline Bandwidth & $1 \mathrm{MHz}$ \\
\hline Channel length (L) & 12 \\
\hline
\end{tabular}

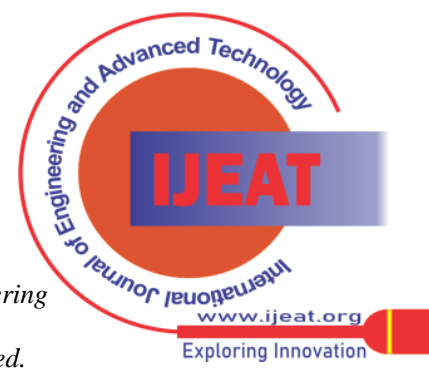


The following assumptions were made in the simulation

a) Normalized Total transmit power

b) Uncorrelated Data symbols

c) Optimum receiver (ML)

d) 4 OFDM symbols per frame

e) Statistically independent Multipath channels for the different pathways

f) Perfect Time and frequency synchronization

g) Guard interval greater than maximum delay spread. h) Simulations for different signal to noise ratio(Es/N0)

i) COST 207 project models on two test urban environments (Typical and Bad)

Table 2 shows the Power Delay Profile of two test urban environments (Typical Urban and Bad Urban)[22]

Table 2: COST Power Delay Profile of two test urban environments

\begin{tabular}{|c|c|c|c|c|c|}
\hline \multicolumn{3}{|c|}{ Urban Environment(Typical) } & \multicolumn{3}{c|}{ Urban Environment(Bad) } \\
\hline Delay [us] & Fractional power & $\begin{array}{c}\text { Doppler } \\
\text { category }\end{array}$ & Delay [us] & $\begin{array}{c}\text { Fractional } \\
\text { power }\end{array}$ & $\begin{array}{c}\text { Doppler } \\
\text { category }\end{array}$ \\
\hline 0.0 & 0.092 & CLASS & 0.0 & 0.033 & CLASS \\
\hline 0.1 & 0.115 & CLASS & 0.1 & 0.089 & CLASS \\
\hline 0.3 & 0.231 & CLASS & 0.3 & 0.141 & CLASS \\
\hline 0.5 & 0.127 & CLASS & 0.7 & 0.194 & GAUS1 \\
\hline 0.8 & 0.115 & GAUS1 & 1.6 & 0.114 & GAUS1 \\
\hline 1.1 & 0.074 & GAUS1 & 2.2 & 0.052 & GAUS2 \\
\hline 1.3 & 0.046 & GAUS1 & 3.1 & 0.035 & GAUS2 \\
\hline 1.7 & 0.074 & GAUS1 & 5.0 & 0.140 & GAUS2 \\
\hline 2.3 & 0.051 & GAUS2 & 6.0 & 0.136 & GAUS2 \\
\hline 3.1 & 0.032 & GAUS2 & 7.2 & 0.041 & GAUS2 \\
\hline 3.2 & 0.018 & GAUS2 & 8.1 & 0.019 & GAUS2 \\
\hline 5.0 & 0.025 & GAUS2 & 10.0 & 0.006 & GAUS2 \\
\hline
\end{tabular}

The BER performance under Typical urban channel model for a 4 QAM SM OFDM system is shown in Fig.5.Low pass estimated channel with DFT came near to that of perfect channel within 2.3 SNR for BER of $10^{-}$ ${ }^{6}$.Error floor happens as SNR increases for linear interpolation. Spline interpolation and low pass interpolation performance remain almost the same. Out of the three interpolation techniques specified, low pass interpolation with DFT performs better when compared to Spline and linear interpolation. In all the three cases,DFT based interpolation performs better with a reduction in noise error.

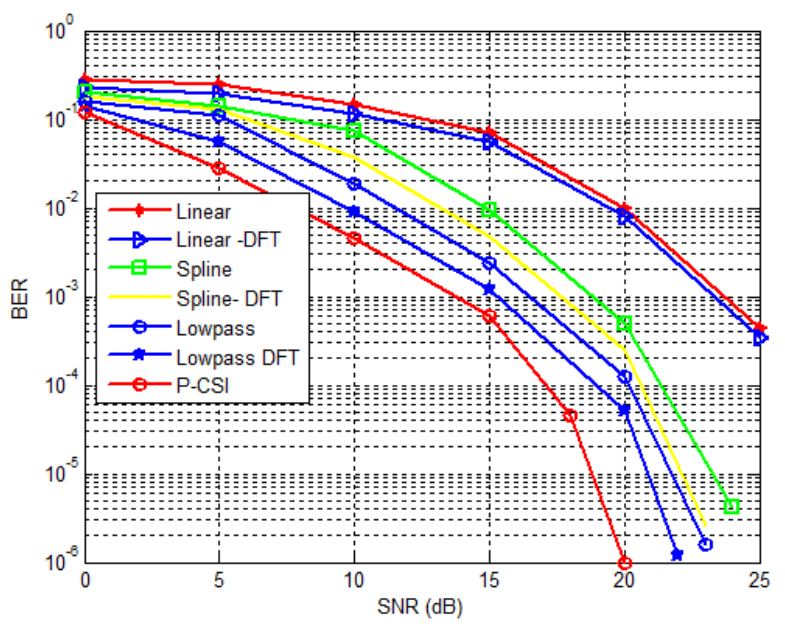

Figure5 BER performance of 4 QAM SM-OFDM under Typical Urban channel model

The BER performance under Bad Urban channel model for a 4 QAM SM OFDM system is shown in Fig.6. The
BER performance remains almost the same as that of a Typical Urban modeland the same conclusion can be drawn.

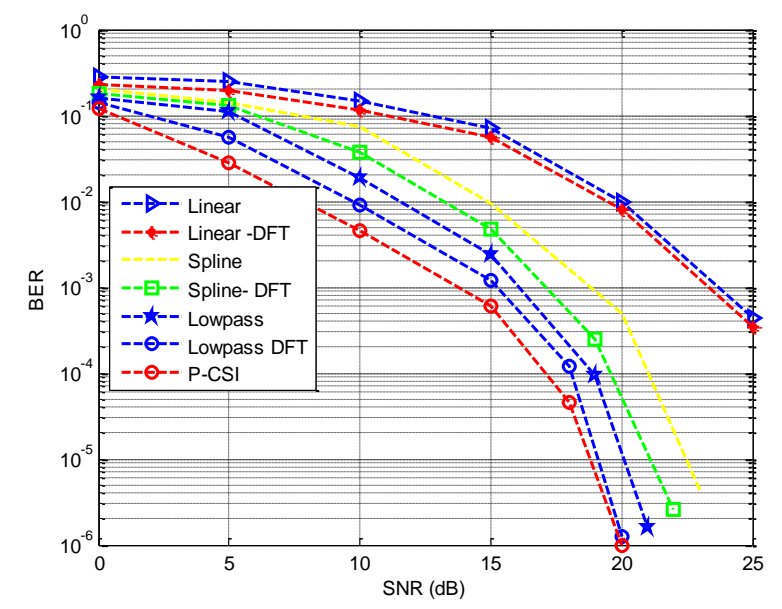

Figure 6 BER performance of 4 QAM SM-OFDM under Bad Urban channel model

Fig.7 and Fig.8 shows BER performance graph of 16 QAM SM OFDM under two channel models. As the constellation size increases, error floor happens to linear interpolation and low pass interpolation with DFT outperforms when compared to other interpolation techniques. Low pass and Spline with DFT remains robust for frequency selective channels.

Published By:

Blue Eyes Intelligence Engineering and Sciences Publication

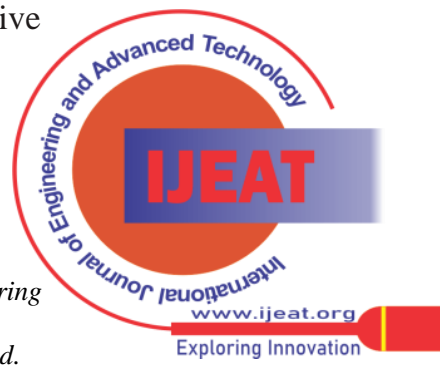




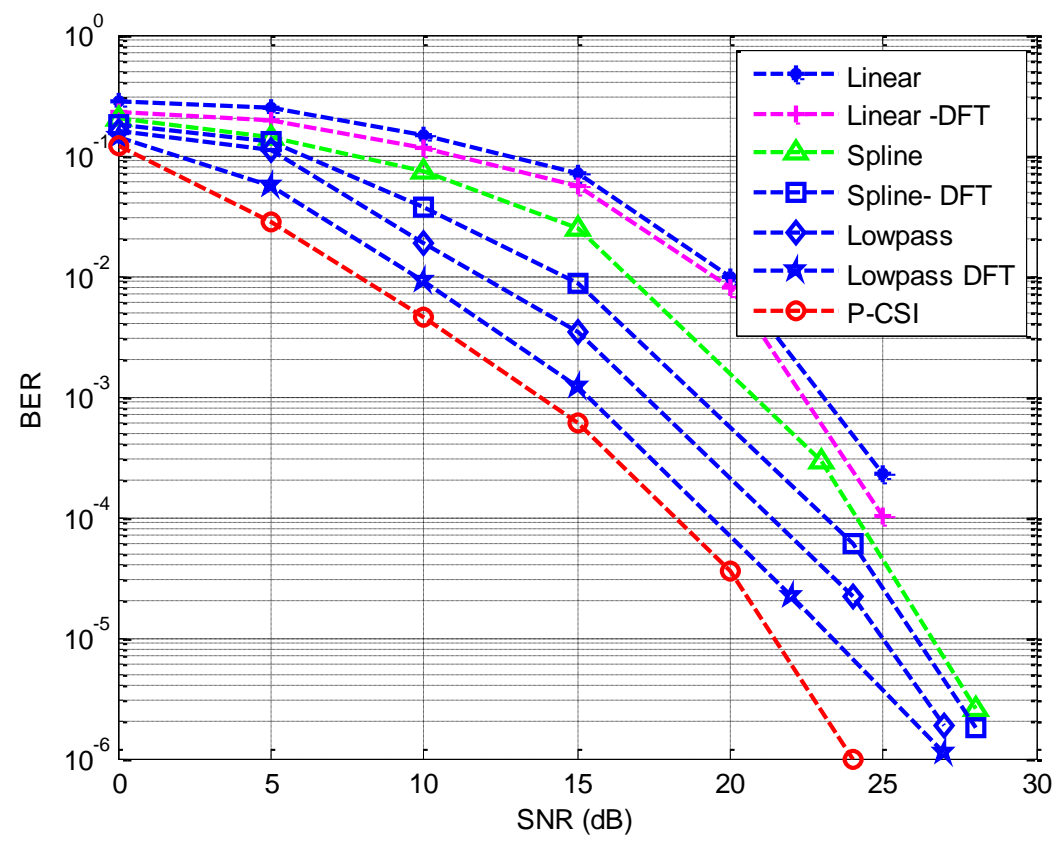

Figure 7 BER performance of 16 QAM SM OFDM under Typical Urban channel model

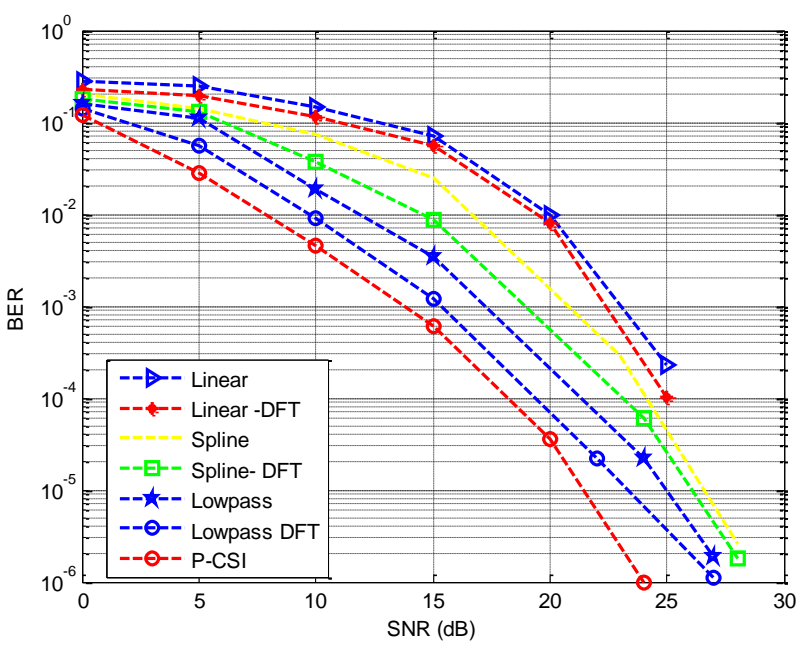

Figure 8 BER performance of 16 QAM SM OFDM under Bad Urban channel model

\section{CONCLUSION}

This paper addressed the channel estimation process of the SM OFDM system. Various details of employing interpolation techniques in the channel estimation process are mainly focused. Results show that the Least Square estimator with DFT based interpolation performs finest of all one dimensional interpolation methods with less computational complexity by employing FFT algorithms. Out of the $1 \mathrm{D}$ interpolation techniques considered, Low pass interpolation with DFT outperforms when compared to others. The performance differences among the interpolation techniques vary according to the coherence bandwidth of the channel. It works effectively when channel delays are integer multiples of sampling time. Incorporation of DFT algorithm enhances BER performance by eliminating the noise effect outside the maximum channel delay length.

\section{REFERENCES}

1. Chiani, M., Win, M. Z., \&Zanella, A. 2003. On the capacity of spatially correlated MIMO Rayleigh-fading channels. IEEE Transactions on Information Theory, 49(10), 2363-2371.

2. Bolcskei, H. 2006. MIMO-OFDM wireless systems: basics, perspectives, and challenges. IEEE wireless communications, 13(4), 31-37.

3. Shiu, D. S., Foschini, G. J., Gans, M. J., \& Kahn, J. M. 2000. Fading correlation and its effect on the capacity of multielement antenna systems. IEEE Transactions on communications, 48(3), 502-513.

4. Loyka, S., \&Tsoulos, G. 2002. Estimating MIMO system performance using the correlation matrix approach. IEEE Communications Letters, 6(1), 19-21.

5. Chiani, M., Win, M. Z., \&Zanella, A.2003. On the capacity of spatially correlated MIMO Rayleigh-fading channels. IEEE Transactions on Information Theory, 49(10), 2363-2371.

6. Mesleh, R. Y., Haas, H., Sinanovic, S., Ahn, C. W., \& Yun, S. 2008. Spatial modulation. IEEE Transactions on vehicular technology, 57(4), 2228-2241.

7. Mesleh, R., Haas, H., Ahn, C. W., \& Yun, S. 2006, October. Spatia modulation-a new low complexity spectral efficiency enhancing technique. In 2006 First International Conference on Communications and Networking in China (pp. 1-5). IEEE.

8. Jeganathan, J., Ghrayeb, A., \&Szczecinski, L. 2008. Spatial modulation: Optimal detection and performance analysis. IEEE Communications Letters, 12(8), 545-547.

9. Acar, Y., Doğan, H., \&Panayırcı, E. 2015. On channel estimation for spatial modulated systems over time-varying channels. Digital Signal Processing, 37, 43-52.

10. Wu, X., Claussen, H., Di Renzo, M., \& Haas, H. 2014. Channel estimation for spatial modulation. IEEE Transactions on Communications, 62(12), 4362-4372.

11. Biguesh, M., \& Gershman, A. B. 2006. Training-based MIMO channel estimation: a study of estimator tradeoffs and optimal training signals. IEEE transactions on signal processing, 54(3), 884893.

12. Yalcin, M., Akan, A., \&Doğan, H. 2012. Low-complexity channel estimation for OFDM systems in high-mobility fading channels. Turkish Journal of Electrical Engineering \& Computer Sciences, 20(4), 583-592.

Blue Eyes Intelligence Engineering and Sciences Publication

(C) Copyright: All rights reserved.

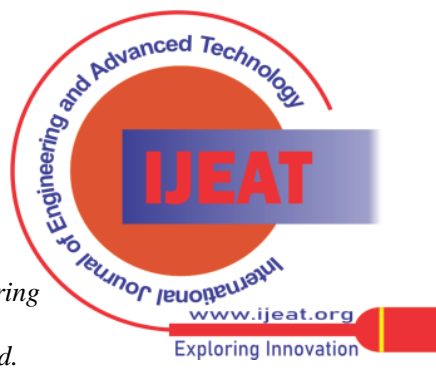


13. Larsson, E. G., \& Li, J. 2001. Preamble design for multiple-antenna OFDM-based WLANs with null subcarriers. IEEE Signal Processing Letters, 8(11), 285-288.

14. Kang, Y., Kim, K., \& Park, H. 2007. Efficient DFT-based channel estimation for OFDM systems on multipath channels. IET communications, 1(2), 197-202.

15. Acar, Y., Doğan, H., \&Panayirci, E. 2017. Pilot symbol aided channel estimation for spatial modulation-OFDM systems and its performance analysis with different types of interpolations. Wireless Personal Communications, 94(3), 1387-1404.

16. Coleri, S., Ergen, M., Puri, A., \& Bahai, A. 2002. Channel estimation techniques based on pilot arrangement in OFDM systems. IEEE Transactions on broadcasting, 48(3), 223-229.

17. Diallo, M., Boher, L., Rabineau, R., Cariou, L., \&Helard, M. 2008, October. Transform domain channel estimation with null subcarriers for MIMO-OFDM systems. In 2008 IEEE International Symposium on Wireless Communication Systems (pp. 209-213). IEEE.

18. Dyer, S. A., \& He, X. 2001. Cubic-spline interpolation: Part 2. IEEE Instrumentation \& Measurement Magazine, 4(2), 34-36.

19. Kahaner, D., Moler, C., \& Nash, S. 1989. Numerical methods and software (p. 1). Englewood Cliffs:Prentice Hall.

20. Fritsch, F. N., \& Carlson, R. E. 1980. Monotone piecewise cubic interpolation. SIAM Journal on Numerical Analysis, 17(2),238-246.

21. Colieri, S., Ergen, M., Puri, A., \& Bahai, A. 2002. A study of channel estimation in OFDM systems.Proceedings the IEEE 56th Vehicular Technology Conference (VTC2002-Fall), (pp. 894-898), (2011).

22. Patzold, M. 2003. Mobile fading channels. Hoboken: Wiley.

\section{AUTHORS PROFILE}

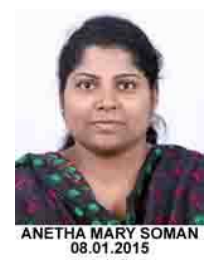

Anetha Mary Soman, is a PhD Student at Pondicherry Central University, Pondicherry,India and an associate professor in Electronics and Communication Engineering Department of St.Thomas College of Engineering \& Technology, Kannur, Kerala, India. She received $\mathrm{BE}$ degree in Electronics and Communication Engineering from Bangalore University and M.Tech in Digital Electronics and Communication from Visveswaraiah Technological University. Her research interest is in wireless communication, digital electronics and signal processing.

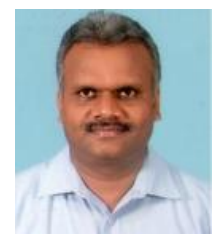

professional

Nakkeeran Rangaswamy, received Ph.D. degree from Pondicherry University in 2004.He is currently working as Associate Professor and Head in the Department of Electronics Engineering, Pondicherry University, Pondicherry ,India. His research interests include antennas, Optical Communications and Wireless Communications. $\mathrm{He}$ is holding

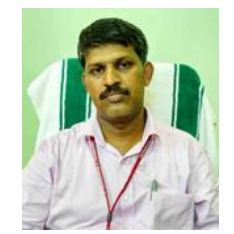
department of Computer Science and Engineering with an experience in the field of Software, Teaching and Research. He received M. E. and B.E. degrees in Computer Science and Engineering from Anna University, Chennai, India and Manonmaniam

Sundaranar University, Tirunelveli, India respectively. He received $\mathrm{PhD}$ degree from Anna University, Chennai, India. He is a member of the System Software Research Group at Anna University Research Centre, Tiruchirappalli. He published papers in various national and international journals and conferences with Scopus and Science Citation index. He is an active reviewer of various indexed journals and his research interests include Grid Computing, Mobile Computing and Computer Networks. Currently he is research supervisor under Kerala Technological University(APJ KTU) .He is a life member of the Indian Society for Technical Education (ISTE), Institution of Engineers ,Computer Society of India(CSI) and a member of IEEE since 2006.

Blue Eyes Intelligence Engineering and Sciences Publication

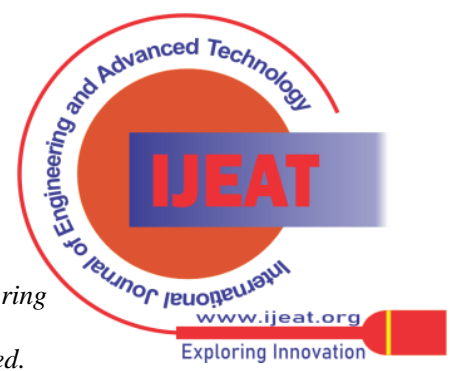

Exploring Innovation 